number of patients reported a willingness to try an online group, we trialled a six-week online Mindfulness and Meditation group.

Aim(s) To discuss the principles of mindfulness and how it can benefit those living with life-limiting illnesses; deliver tangible experience of mindfulness practice; and provide social connectedness to patients with symptoms of anxiety shielding in their homes.

Methods November - December 2020: course scoping and planning; December 2020 - January 2021: patient recruitment; 27 January - 3 March 2021: course delivered via presentation slides on Zoom and weekly homework tasks; March - April 2021: evaluation and service user feedback. Outcome measures: number of attendees, service user feedback.

Results Attendee numbers: 11 patients signed up with between five and eight attending each session. Six patients provided feedback: 100\% reported the course helped them a) feel less stressed and overwhelmed, b) change the way they view their thoughts, and c) change their behaviours. $83 \%$ were either 'satisfied' or 'very satisfied' with the course.

Conclusions The online Mindfulness and Meditation group had good and regular attendance, and service users indicated that it contributed to positive changes in their mental health. The course will run online again in July 2021, this time with pre- and post-questionnaires to assess the reduction in symptoms of anxiety more robustly.

\section{P-59 GROWING CONNECTIONS}

Lucy Heaps. Garden House Hospice Care, Letchworth Garden City, UK

10.1136/spcare-2021-Hospice.77

Background In March 2020, due to COVID-19 the Social and Therapeutic Horticulture (STH) group in day services had to close. The hospice occupational therapist (OT) wanted to support the group to stay connected to nature and each other, so decided to introduce an interactive monthly newsletter.

Aims To encourage and support patients and volunteers to connect with nature (and each other) to support their mental health and sense of wellbeing through a period of shielding and isolation.

Approach

- April 2020 - monthly newsletters were posted to patients and volunteers.

- June 2021 - reviewed and agreed plans to relaunch the STH face-to-face groups (following the social distancing and PPE requirements).

- August 2021 - face-to-face 'wellbeing through nature' sessions to start and newsletters to continue.

Results 32 people have accessed the monthly newsletter, whereas the face-to-face session only had six per session (on average). It has reached patients who had been unable to attend sessions at the centre due to logistics and care needs. It has enabled new patients to connect and benefit. It has kept people connected as they shared their news and stories together in the 'grapevine' section of the newsletter. The OT has inspired good practice for other health professionals.

Conclusion The newsletter has proven to be a fantastic medium for patients and volunteers to stay connected to the hospice and their peers through the pandemic. It has gathered a great deal of positive feedback from patients and families/ carers alike. It has enabled the service to demonstrate that even through the pandemic we have been able to keep to our hospice core values. The newsletter will continue post COVID-19. It has enabled our patients to connect in a virtual way. We now look forward to restarting our face-to-face STH sessions in our day service.

\section{P-60 COMPTON VS COVID: STEPPING UP}

Beverley Clarke, Tricia Evans, Jenny Warren, Angela Matthews, James Elliott. Compton Care, Wolverhampton, UK

\subsection{6/spcare-2021-Hospice.78}

Background and Aims In March 2020 our hospice was faced with an unprecedented challenge. The COVID-19 pandemic affected every aspect of our work, from patient care to fundraising, but proactive cooperation with local services enabled us to 'Step Up' our support for patients and families.

Method and Results The 'Stepping Up' appeal found innovative ways to raise funds and engage with the public, from virtual events to gathering messages of support for our hardworking staff. Our Compassionate Communities team launched a telephone befriending service and virtual bereavement information hubs, reaching out to those isolated by the pandemic. Our inpatient unit expanded to better facilitate admissions from local hospitals and the community, overseen by a dedicated flow co-ordinator. The team was rapidly upskilled in managing more acutely unwell patients, including those with COVID-19, and challenging situations such as withdrawal of NIV.

Our Living Well Centre, temporarily closed due to the pandemic, was transformed into a nurse-led unit for COVID-19positive patients awaiting care home placement. As well as being redeployed, our Living Well nurses embraced new technology to support their patients remotely. Our community team provided additional support to local district nursing teams, taking over personal care for some of their more complex palliative patients. Training in procedures such as catheter insertion and venepuncture was also expedited. Working collaboratively with NHS colleagues we were able to manage more complex medical situations at home, reducing the need for inpatient admissions. Video consultations and a professional support line allowed the medical team to become more responsive.

Conclusion Despite the many challenges, we have taken the opportunity to improve continuity of care and build better working relationships within our organisation. Cooperation with external partners, a keystone of good palliative care, has been vital to our ability to respond and adapt, and we hope to see these positive changes continue.

\section{P-61 ST LEONARD'S HOSPICE COVID-19 RESPONSE - COORDINATION AND RESPONSE}

Jenny Brandom. St Leonard's Hospice, York, UK

\subsection{6/spcare-2021-Hospice. 79}

Background St Leonard's Hospice were approached by the local health and care system to lead the response and co-ordination of care for those people requiring end-of-life care in 